\title{
Nonelective surgery at night and in-hospital mortality
}

\section{Prospective observational data from the European Surgical Outcomes Study}

\author{
Bas van Zaane, Wilton A. van Klei, Wolfgang F. Buhre, Peter Bauer, E. Christiaan Boerma, \\ Andreas Hoeft, Philipp Metnitz, Rui P. Moreno, Rupert Pearse, Paolo Pelosi, Michael Sander, \\ Benoit Vallet, Ville Pettilä, Jean-Louis Vincent and Andrew Rhodes, for the European Surgical \\ Outcomes Study (EuSOS) group for the Trials groups of the European Society of Intensive Care \\ Medicine and the European Society of Anaesthesiology*
}

\begin{abstract}
BACKGROUND Evidence suggests that sleep deprivation associated with night-time working may adversely affect performance resulting in a reduction in the safety of surgery and anaesthesia.
\end{abstract}

OBJECTIVE Our primary objective was to evaluate an association between nonelective night-time surgery and inhospital mortality. We hypothesised that urgent surgery performed during the night was associated with higher inhospital mortality and also an increase in the duration of hospital stay and the number of admissions to critical care.

DESIGN A prospective cohort study. This is a secondary analysis of a large database related to perioperative care and outcome (European Surgical Outcome Study).

SETTING Four hundred and ninety-eight hospitals in 28 European countries.

PATIENTS Men and women older than 16 years who underwent nonelective, noncardiac surgery were included according to time of the procedure.

INTERVENTION None.

MAIN OUTCOME MEASURES Primary outcome was inhospital mortality; the secondary outcome was the duration of hospital stay and critical care admission.
RESULTS Eleven thousand two hundred and ninety patients undergoing urgent surgery were included in the analysis with 636 in-hospital deaths (5.6\%). Crude mortality odds ratios (ORs) increased sequentially from daytime [426 deaths $(5.3 \%)$ ] to evening [150 deaths (6.0\%), OR $1.14 ; 95 \%$ confidence interval 0.94 to 1.38 ] to night-time [60 deaths (8.3\%), OR 1.62; 95\% confidence interval 1.22 to 2.14 ]. Following adjustment for confounding factors, surgery during the evening (OR 1.09; 95\% confidence interval 0.91 to 1.31 ) and night (OR 1.20; 95\% confidence interval 0.9 to 1.6) was not associated with an increased risk of postoperative death. Admittance rate to an ICU increased sequentially from daytime [891 (11.1\%)], to evening [347 (13.8\%)] to night time [149 (20.6\%)].

CONCLUSION In patients undergoing nonelective urgent noncardiac surgery, in-hospital mortality was associated with well known risk factors related to patients and surgery, but we did not identify any relationship with the time of day at which the procedure was performed.

TRIAL REGISTRATION Clinicaltrials.gov identifier: NCTO 1203605

From the Department of Anaesthesiology, University Medical Centre Utrecht, Utrecht (BVZ,WAVK), Department of Anaesthesiology, Maastricht University Medical Centre, Maastricht, the Netherlands (WFB), Section of Medical Statistics, Medical University of Vienna, Vienna, Austria (PB), Department of Intensive Care, Medical Centre Leeuwarden, Leeuwarden, the Netherlands (ECB), Department of Anaesthesiology, University of Bonn, Bonn, Germany (AH), Department of General Anaesthesiology, Emergency and Intensive Care Medicine, Medical University of Graz, Graz, Austria (PM), UCINC, Hospital de São José, Centro Hospitalar de Lisboa Central, EPE, Lisbon, Portugal (RPM), Barts and The London School of Medicine and Dentistry, Queen Mary University of London, Department of intensive care, London, UK (RP), IRCCS AOU San Martino-IST (RP), Department of Surgical Sciences and Integrated Diagnostics, University of Genoa, Genova, Italy (PP), Charité-Universitaetsmedizin, Department of Anaesthesiology (MS), Anaesthesiology and Critical Care, University Hospital Lille, Lille, France (BV), Intensive Care Units, Helsinki University Hospital, Helsinki, Finland (VP), Erasme Hospital, Université Libre de Bruxelles, Brussels, Belgium (J-LV), and St George's Healthcare NHS Trust, London, UK (AR)

Correspondence to Bas van Zaane, Department of Anesthesiology, University Medical Centre Utrecht, Mailstop: Q.04.2.313, P.O. Box 85500, Utrecht 3508 GA, the Netherlands

Tel: +31 8875 55555; e-mail: b.vanzaane@umcutrecht.nl

*Members listed in appendix I, http://links.Iww.com/EJA/A68. 


\section{Introduction}

Over 230 million surgical procedures are performed worldwide each year with an estimated mortality between 1 and 4\%. ${ }^{1,2}$ Mortality following noncardiac surgery may be higher than previously thought and may partly relate to high mortality rates following emergency surgery. $^{2-5}$ The factors contributing to mortality following surgery are complex and may include the urgency of surgery, the availability and performance of personnel and the time of day.

The findings of several studies investigating various medical procedures have suggested an association between medical care administered at night and increased morbidity and mortality. ${ }^{6-8}$ Patients requiring emergency surgery are at an increased risk compared with elective surgery, even when the procedure is performed under ideal circumstances. 9,10 If such procedures are performed during the evening or night, the outcome may be adversely affected, as the most skilled professionals are not always immediately available or their performance may be influenced by physical or mental fatigue. It is known that fatigue increases the incidence of medical error, ${ }^{11}$ making it one of the reasons why it is thought safer to undertake surgery during the day-time whenever possible. ${ }^{12-14}$

As far as we know, this possible association between time point of operation and mortality following emergency surgery has not been studied before. Therefore, the aim of this study was to investigate, in the European Surgical Outcomes Study (EuSOS) database, the association between the time of the day (daytime, evening, nighttime) at which urgent surgery is performed, and inhospital mortality after surgery. ${ }^{2}$ We hypothesised that urgent surgery performed during the night was associated with higher in-hospital mortality and also longer hospital stays and more critical care admissions.

\section{Materials and methods}

Data were taken from the European Surgical Outcomes Study (EuSOS) database, the methods of which are described in detail elsewhere. ${ }^{2,15}$ In brief, the European cohort study was performed between 09:00 (local time) on 4 April 2011, and 08:59 on 11 April 2011. All patients older than 16 years admitted to participating centres for elective or nonelective inpatient surgery commencing during the 7-day period were eligible for inclusion. Patients undergoing planned day-case surgery, cardiac surgery, neurosurgery, radiological or obstetric procedures were excluded. Participating hospitals were a voluntary convenience sample, identified through membership of the European Society of Intensive Care Medicine and the European Society of Anaesthesiology and by direct approach from national study coordinators.

\section{Ethics}

Ethical approval for this study (Ethical Committee Number $10 / \mathrm{H} 0605 / 72$ ) was primarily provided by the ethical committee of Southampton University Hospitals NHS trust, Southampton, United Kingdom, on the 15 November 2010. Subsequently, each of the 498 participating centres applied for ethical approval. The requirements differed by country. In Denmark, centres were exempt from ethics approval because this study was technically a clinical audit. In all other nations, formal ethics approval was applied for and given. In Finland, we were required to obtain written informed consent from individual patients.

\section{Cohort description}

For this secondary analysis, all patients within the EuSOS database who had urgent or emergency surgery were included. Exclusion criteria were any site enrolling fewer than 10 patients in the study week, any site having an in-hospital mortality rate either above or below the 95th centile, any patient with missing data for in-hospital mortality, any patient with missing data for the urgency of surgery and any patient presenting for an elective surgical procedure. In EuSOS, the definition of emergency surgery was immediately, without delay, ideally within $24 \mathrm{~h}$. The definition of urgent surgery was planned surgery within hours or days of the decision to operate. The severity of surgery indicated a combination of complexity and amount of tissue injury. Minor surgery would include procedures that would often involve extremities or the body surface lasting less than $30 \mathrm{~min}$ performed in a dedicated operating room, brief diagnostic and therapeutic procedures such as arthroscopy without intervention and removal of small cutaneous tumours. Intermediate procedures were more prolonged or complex with the risk of significant complication or tissue injury. Examples might include laparoscopic cholecystectomy, arthroscopy with intervention and fixation of a mandibular fracture. Major surgical procedures were expected to last more than $90 \mathrm{~min}$ and included major gut resection, major joint replacement, mastectomy, extensive head and neck tumour resection, abdominal aortic aneurysm repair and major vascular bypass procedure.

\section{Timing of surgery}

Patients within the cohort were stratified by the time of surgery using time of induction of anaesthesia as the starting time of the procedure. A dummy variable 'time of day' was constructed that was defined as 'daytime' if between 08:01 and 17:00, 'evening' if between 17:01 and 24:00, and 'night-time' if between $00: 01$ and $08: 00$. 


\section{Outcomes}

The primary outcome was in-hospital mortality; the secondary outcome was the number of critical care admissions.

\section{Statistical analysis}

We used SPSS (version 19.0) for data analysis. Categorical variables are presented as number $(\%)$ and continuous variables as mean (SD) when normally distributed or median (interquartile range, IQR) when not. We used $\chi^{2}$ and Fisher's exact tests to compare categorical variables and the $t$-test or the Mann-Whitney $U$ test to compare continuous variables. For categorical variables describing differences in hospital characteristics across time, a logistic regression analysis was used with the site being entered as a random factor. Significance was set at a $P$ value less than 0.05 . As the rate of missing values was very low $(<0.05 \%)$, no imputation procedures were performed.

Logistic multilevel regression analysis was used to determine whether or not in-hospital mortality was different depending on the time of day the procedure occurred, as depicted by the hour of day anaesthesia commenced, and to adjust for identified confounding factors affecting outcome. The first step was to identify factors that were independently related to in-hospital mortality from univariable analysis. The following factors were entered into the model on the basis of their univariable relationship to outcome: age, American Society of Anesthesiologists (ASA) status as recorded just prior to induction ${ }^{16}$ (reference category ASA I), urgency of surgery (reference urgent), severity of surgery (reference minor) and the presence of cirrhosis. Due to the multiplicity of tests performed and in order to avoid spurious associations and overfitting, only $P$ values of less than 0.01 were considered as significant and therefore included in the model in order to allow for a consistent result. All factors entered were selected according to their scientific plausibility, and a low rate of missing data across the whole sample. The results of the model were reported as odds ratios (ORs) with 95\% confidence interval (95\% CI). We assessed the models through sensitivity analyses with four random (disjoint) subsamples of the cohort. The findings from the multivariable analysis were validated in a number of predefined subgroups, that is university hospital status, age less than 80 years, emergency procedures, intra-abdominal procedure and nonconsultant anaesthetist/surgeon performing procedure.

The second step was to evaluate the effects of time of day for the procedure. This was performed by constructing a subsequent model using the same inference factors as above, but including the time of day as a covariable to investigate a potential trend using daytime as the reference. The individual hospital was entered into each analysis as a random factor in order to adjust for the clustering effect with the consequent breaking of the assumption that all observations are independent of one another. The analysis was repeated with time included per hour. Finally, we repeated the analysis with all hospitals, and patients.

\section{Sample size}

As the current study was a retrospective secondary analysis on the EuSOS database, we did not perform a formal sample size calculation in advance. In general, it is considered that one can include one confounder (or variable) for every 10 cases in a multivariable regression model. ${ }^{17}$ With a total of 636 deaths in our database, we were able to adjust for all relevant confounders.

\section{Results}

Of the 46539 unique patients included in the original EuSOS database, 11290 (24.3\%) patients fulfilled the cohort criteria and were included in this study (Fig. 1). Characteristics of enrolling hospitals are presented in Table 1. Of the 11290 patients, 8055 (71\%), 2510 $(22 \%)$ and $725(6 \%)$ underwent urgent or emergency surgery during either the day, evening or night, respectively. Baseline characteristics of the patients are presented in Table 2 divided according to the time of day the procedure was performed. The majority underwent orthopaedic surgery or surgery of the lower gastro-intestinal tract. Patients having surgery at night were younger, more frequently received general anaesthesia and were more frequently graded ASA III, IV or V. Unadjusted 30day in-hospital mortality for patients who underwent urgent or emergency surgery was $5.6 \%$. The overall inhospital mortality in patients who underwent emergency or urgent surgery was 9.1 and $4.2 \%$, respectively.

In-hospital mortality after emergency or urgent surgery was $426(5.3 \%), 150(6.0 \%)$ and $60(8.3 \%)$ during the day, the evening or the night, respectively. Night-time surgery was associated with a significantly higher inhospital mortality rate than day-time surgery with an unadjusted OR of 1.62 (95\% CI 1.22 to 2.14, $P=0.001)$. Other associations with in-hospital mortality included higher ASA grades, upper gastrointestinal surgical procedures, major emergency surgery and the presence of comorbid diseases. The highest in-hospital mortality was found in patients who underwent urgent or emergency surgery between 4:00 and 6:59, with a peak incidence of $19.1 \%$, and an OR of 6.37 (95\% CI 2.72 to 14.95 ) between $4: 00$ and 4:59 (Fig. 2). Patients who underwent urgent or emergency surgery during the night had a higher probability of being admitted to a critical care unit. Eight hundred and ninety-one (11.1\%), 347 $(13.8 \%)$ and $149(20.6 \%)$ patients were admitted to an ICU after day, evening and night procedures, respectively. Admittance to an ICU was planned for $483(54.3 \%)$ patients during the day, for $137(39.7 \%)$ patients during the evening and for $54(36.2 \%)$ patients during the night. 
Fig. 1

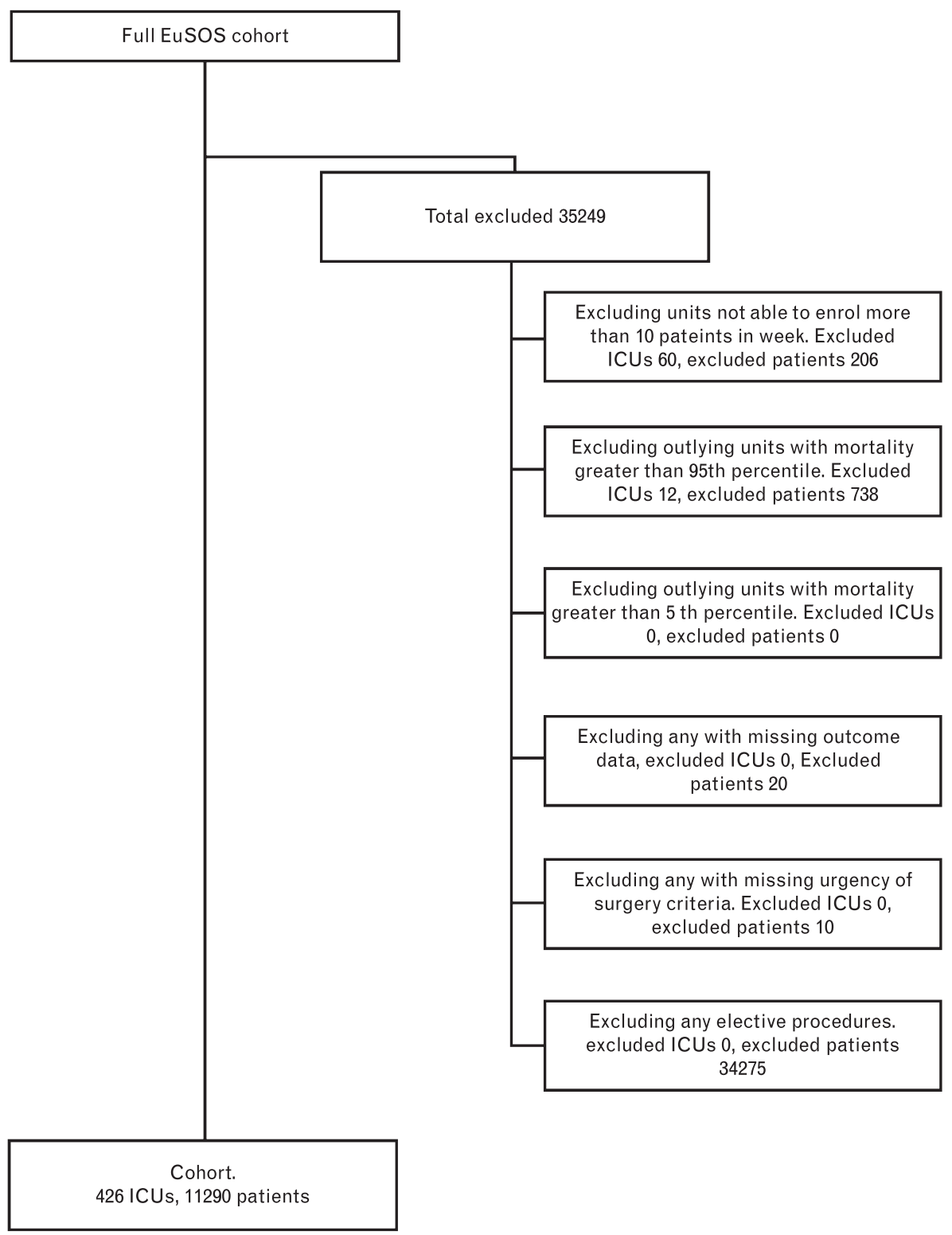

Description of cohort.

Table 1 Hospital characteristics and overall outcomes according to the time of operative procedure

\begin{tabular}{|c|c|c|c|c|}
\hline & Day $08: 01$ to $17: 00$ & Evening $17: 01$ to $24: 00$ & Night $00: 01$ to $08: 00$ & $\boldsymbol{P}$ \\
\hline No. (\%) from University centre & $5292 / 7992(66.2)$ & 1707/2494 (68.4) & $547 / 720(76.0)$ & 0.001 \\
\hline No. of operating theatres & 14 (9 to 21$)$ & 15 (9 to 22$)$ & $17(10$ to 25$)$ & $<0.001$ \\
\hline No. high dependency beds & $6(0$ to 11$)$ & 6 (0 to 12$)$ & $6(0$ to 12$)$ & 0.21 \\
\hline No. of intensive care beds & 12 (8 to 24$)$ & 14 (9 to 28 ) & $18(10$ to 32$)$ & $<0.001$ \\
\hline No. (\%) admitted to critical care & $891 / 8053(11.1)$ & $347 / 2510(13.8)$ & $149 / 725(20.6)$ & $<0.001$ \\
\hline Length hospital of stay (days) & $4(2$ to 10$)$ & $4(2$ to 10$)$ & $4(1$ to 10$)$ & 0.01 \\
\hline In-hospital mortality (\%) & $426 / 8055(5.3)$ & $150 / 2510(6.0)$ & $60 / 725(8.3)$ & 0.003 \\
\hline Duration of surgery ( $\mathrm{min}$ ) & 90 (60 to 135$)$ & $80(52-120)$ & 90 (58 to 130$)$ & $<0.001$ \\
\hline
\end{tabular}

Figures are given as median (IOR) except where indicated (\%). 
Table 2 Baseline characteristics according to the time of operative procedure

\begin{tabular}{|c|c|c|c|c|}
\hline & $\begin{array}{l}8: 01 \text { to } 17: 00 \\
n=8055 \text { Day }\end{array}$ & $\begin{array}{l}\text { Evening } 17: 01 \text { to } 24: 00 \\
\qquad n=2510\end{array}$ & $\begin{array}{l}\text { Night } 00: 01 \text { to } 08: 00 \\
n=725\end{array}$ & $\boldsymbol{P}$ \\
\hline Male sex (\%) & $5292(66.2)$ & $1285(51.2)$ & $406(56.0)$ & 0.07 \\
\hline Mean age in years (SD) & $57(22)$ & $53(22)$ & $51(21)$ & $<0.001$ \\
\hline \multicolumn{5}{|l|}{ Anaesthetic technique (\%) } \\
\hline General anaesthesia & $6264(77.8)$ & $1983(79.0)$ & 640 (88.3) & $<0.001$ \\
\hline Spinal anaesthesia & $1353(16.8)$ & $373(14,9)$ & $49(6.8)$ & $<0.001$ \\
\hline Epidural anaesthesia & $249(3.1)$ & $62(2.5)$ & $23(3.2)$ & 0.26 \\
\hline ASA classification (\%) & & & & $<0.001$ \\
\hline 1 & $2086(26.0)$ & 807 (32.2) & $236(32.6)$ & \\
\hline$\|$ & 2768 (34.4) & $879(35.1)$ & $223(30.8)$ & \\
\hline III & 2501 (31.1) & $596(23.8)$ & $175(24.1)$ & \\
\hline IV & $642(8.0)$ & $199(7.9)$ & $73(10.1)$ & \\
\hline $\mathrm{v}$ & $39(0.5)$ & $24(1.0)$ & $18(2.5)$ & \\
\hline Surgical specialty (\%) & & & & $<0.001$ \\
\hline Orthopaedics & 3125 (38.9) & $687(27.5)$ & $105(14.5)$ & \\
\hline Breast & $88(1.1)$ & $11(0.4)$ & $3(0.4)$ & \\
\hline Gynaecology & $379(4.7)$ & $153(6.1)$ & $46(6.4)$ & \\
\hline Vascular & $503(6.3)$ & $116(4.6)$ & $42(5.8)$ & \\
\hline Upper gastrointestinal & $463(5.8)$ & $181(7.3)$ & $64(8.9)$ & \\
\hline Lower gastrointestinal & $1284(16.0)$ & $730(29.3)$ & $269(37.2)$ & \\
\hline Hepato-biliary & $288(3.6)$ & $87(3.5)$ & $38(5.3)$ & \\
\hline Plastic/cutaneous & $430(5.4)$ & $155(6.2)$ & $39(5.4)$ & \\
\hline Urology & $460(5.7)$ & $97(3.9)$ & $31(4.3)$ & \\
\hline Kidney & $68(0.8)$ & $21(0.8)$ & $10(1.4)$ & \\
\hline Head and neck & $471(5.9)$ & $104(4.2)$ & $30(4.1)$ & \\
\hline Other & $467(5.8)$ & $153(6.1)$ & $46(6.4)$ & \\
\hline \multicolumn{5}{|l|}{ Comorbidities (\%) } \\
\hline Cirrhosis & $116(1.4)$ & $38(1.5)$ & $27(3.7)$ & $<0.001$ \\
\hline Congestive heart failure & $547(6.8)$ & $143(5.7)$ & $37(5.1)$ & 0.047 \\
\hline COPD & $967(12.0)$ & $233(9.3)$ & $77(10.7)$ & 0.001 \\
\hline Coronary artery disease & $1.293(16.1)$ & $318(12.7)$ & $75(10.4)$ & $<0.001$ \\
\hline Diabetes mellitus (insulin dependent) & $483(6.0)$ & $125(5.0)$ & $38(5.3)$ & 0.137 \\
\hline Diabetes mellitus (noninsulin dependent) & $602(7.5)$ & $154(6.2)$ & $41(5.7)$ & 0.023 \\
\hline Metastatic cancer & $429(5.3)$ & 89 (3.6) & $23(3.2)$ & $<0.001$ \\
\hline Stroke & $496(6.2)$ & $96(3.8)$ & $31(4.3)$ & $<0.001$ \\
\hline
\end{tabular}

Data are absolute numbers (\%) unless otherwise specified.

The following variables were independently associated with in-hospital death and were thus used to adjust the univariable estimates of mortality: age, ASA status,

Fig. 2

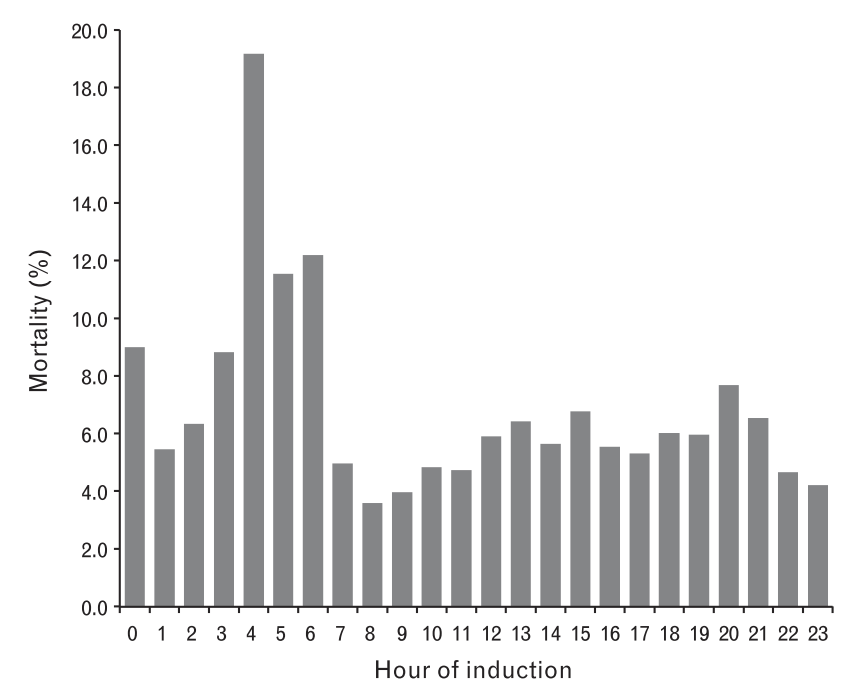

In-hospital mortality (\%) on operated patients by hour of induction. severity of surgical procedure, urgency of surgical procedure and the presence of cirrhosis (Table 3). These variables were entered into a two-level hierarchical multivariable model with hospital as a random factor at the second level. After adjustment for factors associated with mortality in the univariable analysis, neither evening (OR 1.09; 95\% CI 0.91 to $1.31 ; P=0.35$ ) nor night-time surgery (OR $1.20 ; 95 \%$ CI 0.90 to 1.60 ; $P=0.36)$ was significantly associated with increased in-hospital mortality (Table 4). The findings from the multivariable analysis were then validated in a number of predefined subgroups, none of which suggested any association between increased in-hospital mortality rates and surgery taking place during the evening and nighttime. The sensitivity analysis showed good internal validity of the fitted model. In the per hour multivariable analysis, all univariable significant results were no longer significant (Fig. 3). Repeating the analysis with all hospitals included in the analysis did not changed the results.

\section{Discussion}

The principal finding of this study was that despite a higher in-hospital mortality rate for patients who underwent urgent or emergency noncardiac surgery at night, 
Table 3 Univariable binary logistic regression analysis (using hospital mortality as the dependent factor) presented as an odds ratio together with its $95 \%$ confidence interval

\begin{tabular}{|c|c|c|}
\hline & Odds ratio $(95 \% \mathrm{CI})$ & $\boldsymbol{P}$ \\
\hline \multicolumn{3}{|l|}{ Time of surgery } \\
\hline Day & Reference & \\
\hline Evening & $1.14(0.94$ to 1.38$)$ & 0.19 \\
\hline Night & $1.62(1.22$ to 2.14$)$ & 0.001 \\
\hline \multicolumn{3}{|l|}{ ASA } \\
\hline 1 & Reference & \\
\hline 2 & 1.62 (1.14 to 2.29$)$ & 0.007 \\
\hline 3 & 4.76 (3.47 to 6.53$)$ & $<0.0001$ \\
\hline 4 & 20.71 (15.00 to 28.60$)$ & $<0.0001$ \\
\hline 5 & 76.30 ( 45.30 to 128.60$)$ & $<0.0001$ \\
\hline \multicolumn{3}{|l|}{ Surgical procedure } \\
\hline Orthopaedics & 0.56 (0.39 to 0.79$)$ & 0.001 \\
\hline Breast & $0.93(0.38$ to 2.24$)$ & 0.87 \\
\hline Gynaecology & $0.45(0.25$ to 0.80$)$ & 0.007 \\
\hline Vascular & 1.76 (1.18 to 2.62$)$ & 0.005 \\
\hline Upper gastrointestinal & 2.05 (1.40 to 3.02$)$ & $<0.0001$ \\
\hline Lower gastrointestinal & $1.10(0.78$ to 1.57$)$ & 0.58 \\
\hline Hepato-biliary & $1.42(0.89$ to 2.25$)$ & 0.14 \\
\hline Plastic/cutaneous & 0.65 (0.39 to 1.07$)$ & 0.09 \\
\hline Urology & $0.69(0.42$ to 1.14$)$ & 0.14 \\
\hline Kidney & $0.31(0.07$ to 1.29$)$ & 0.11 \\
\hline Head and neck & $0.59(0.35$ to 0.99$)$ & 0.05 \\
\hline Other & Reference & \\
\hline \multicolumn{3}{|l|}{ Urgency of surgery } \\
\hline Urgent & Reference & \\
\hline Emergency & $2.08(1.76$ to 2.46$)$ & $<0.0001$ \\
\hline \multicolumn{3}{|l|}{ Severity of surgery } \\
\hline Minor & Reference & \\
\hline Intermediate & $1.02(0.80$ to 1.31$)$ & 0.88 \\
\hline Major & 3.36 (2.66 to 4.25$)$ & $<0.0001$ \\
\hline \multicolumn{3}{|l|}{ Comorbidities } \\
\hline Cirrhosis & 4.83 (3.36 to 6.95 ) & $<0.0001$ \\
\hline $\begin{array}{l}\text { Chronic obstructive pulmonary } \\
\text { disease }\end{array}$ & 1.68 (1.35 to 2.08$)$ & $<0.0001$ \\
\hline Coronary artery disease & 2.50 (2.09 to 2.99 ) & $<0.0001$ \\
\hline $\begin{array}{l}\text { Diabetes mellitus (insulin } \\
\text { dependent) }\end{array}$ & 1.86 (1.41 to 2.45$)$ & $<0.0001$ \\
\hline Metastatic cancer & 2.72 (2.09 to 3.54$)$ & $<0.0001$ \\
\hline Stroke & $2.14(1.63$ to 2.79$)$ & $<0.0001$ \\
\hline Congestive cardiac failure & 3.53 (2.83 to 4.89$)$ & $<0.0001$ \\
\hline Age (per year) & $1.03(1.03$ to 1.04$)$ & $<0.0001$ \\
\hline
\end{tabular}

this association was no longer observed following adjustment for other known patient and surgery-related risk factors. Our analysis of the EuSOS dataset did not confirm a relationship between the time of day urgent or emergency surgery was performed and postoperative in-hospital death. Patients who were operated on during the night had a higher probability of being admitted to an ICU. The main strengths of this study are that we used a large prospective, multicentre and multinational database, which gave us the opportunity to explore different types of surgery and possible risk factors.

Our findings are comparable to the results of a study on thoracic organ transplant procedures, reporting no significant association between time of day and in-hospital mortality. ${ }^{18}$ The authors of this study hypothesised that personnel involved in transplant surgery had developed alternative systems to cope with the limitations of nighttime work. For example, they suggest that all personnel
Table 4 Hierarchical two-level binary logistic regression model (hospital as a random factor at the second level) assessing preoperative factors relating to in-hospital mortality (probability of death $=1$ ) using a two-level model (patient/hospital) with hospital as a random factor

\begin{tabular}{|c|c|c|}
\hline Variable & OR $(95 \% \mathrm{CI})$ & $\boldsymbol{P}$ \\
\hline \multicolumn{3}{|l|}{ Time of surgery } \\
\hline $08: 00$ to $17: 00$ & 1 & \\
\hline $17: 00$ to $24: 00$ & 1.09 (0.91 to 1.31$)$ & 0.35 \\
\hline $24: 00$ to $08: 00$ & $1.20(0.90$ to 1.60$)$ & 0.22 \\
\hline \multicolumn{3}{|l|}{ ASA } \\
\hline 1 & 1 & \\
\hline 2 & $0.89(0.69$ to 1.17$)$ & 0.42 \\
\hline 3 & $1.37(1.03$ to 1.82$)$ & 0.03 \\
\hline 4 & 4.74 (3.51 to 6.40$)$ & $<0.0001$ \\
\hline 5 & 14.61 (8.71 to 24.49$)$ & $<0.0001$ \\
\hline \multicolumn{3}{|l|}{ Urgency of surgery } \\
\hline Urgent & 1 & \\
\hline Emergency & 1.33 (1.08 to 1.67$)$ & 0.001 \\
\hline \multicolumn{3}{|l|}{ Severity of surgery } \\
\hline Minor & 1 & \\
\hline Intermediate & $0.91(0.74$ to 1.13$)$ & 0.4 \\
\hline Major & $1.34(1.08$ to 1.67$)$ & 0.001 \\
\hline \multicolumn{3}{|l|}{ Comorbidities } \\
\hline Cirrhosis & $2.10(1.41$ to 3.13$)$ & $<0.0001$ \\
\hline Age (per year) & 1.01 (1.01 to 1.020$)$ & $<0.0001$ \\
\hline
\end{tabular}

Included 11 206; excluded 84; Total 11 290.ASA, American Society of Anesthesiologist's Physical Status Class; OR $(95 \% \mathrm{Cl})$ odds ratio (95\% confidence interval).

have extensive experience in these procedures and are less prone to make mistakes even when the procedures are performed during the night. This suggestion should be regarded cautiously. If true, only the most experienced staff (surgeons, anaesthesiologists, operating room staff) should be available during the night, and not the less experienced senior resident or fellow with direct or indirect supervision. In contrast, a number of studies suggest that older doctors are more likely to struggle with the demands of night-time working. ${ }^{19,20}$ There is more evident from a recent study of elective daytime cholecystectomy that compared the rate of conversion with open cholecystectomy, iatrogenic complications and death, according to whether the surgeon had worked the night before or not; no association was found. ${ }^{21}$. Our findings are in contrast with previous studies examining medical care at night. These show that patients undergoing percutaneous coronary interventions for myocardial infarction had a higher incidence of myocardial infarction and death, ${ }^{6}$ emergency orthopaedic surgery was associated with a higher rate of re-exploration, ${ }^{7}$ night-time kidney transplant was associated with an increased risk of graft failure ${ }^{22}$ and end-of-day procedures had more anaesthetic adverse events. ${ }^{23}$ A correction for confounding factors was performed in all above-mentioned studies. These studies probably did not include the same confounders as we did, and therefore, it is difficult to make a direct comparison with our own. Inclusion of confounders merely depends on clinical and scientific reasoning, and is thus based on the researcher's individual considerations. There are a variety of possible explanations for the findings in these studies that include fatigue of the team members, 


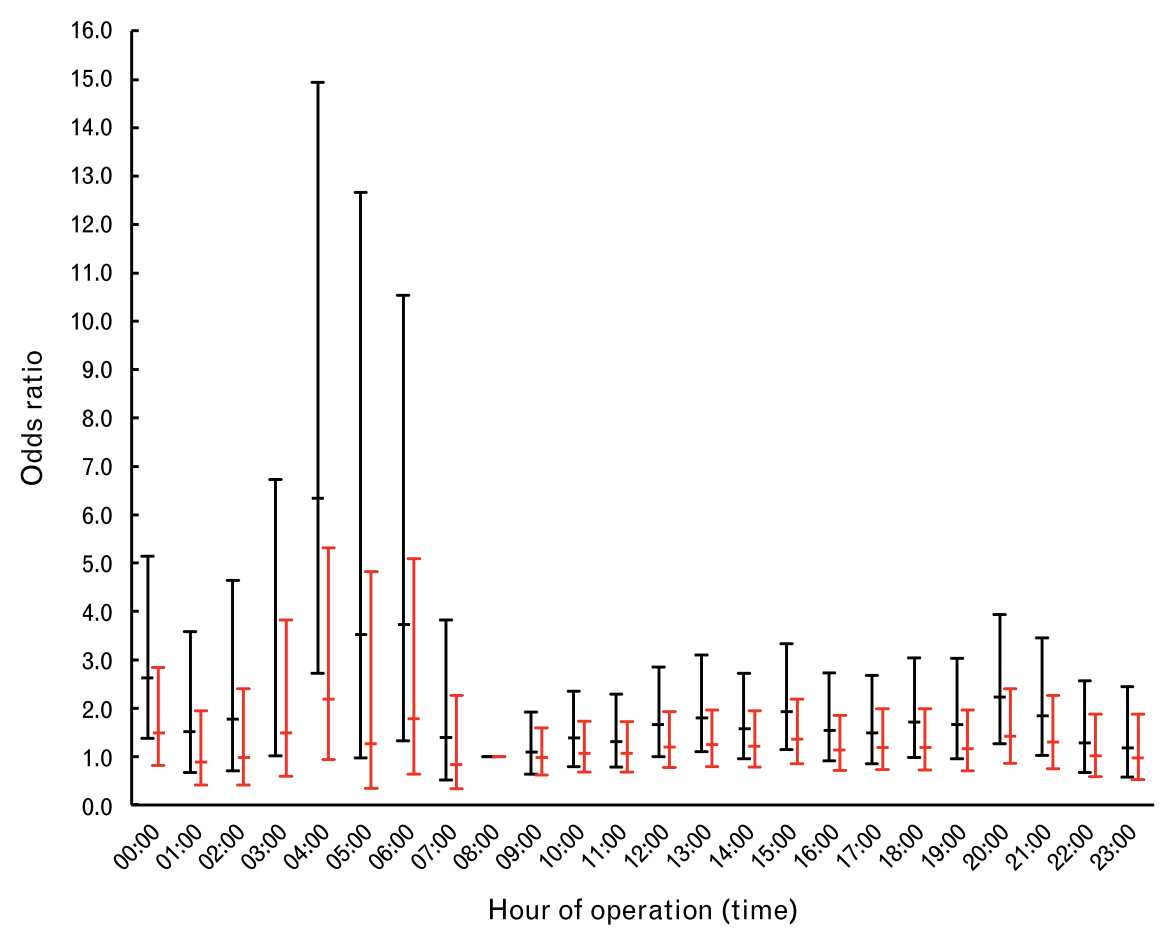

Odds ratios with $95 \%$ confidence interval for in-hospital mortality on operated patients by hour of induction (black, Univariable results; red, multivariable results).

information loss due to shift changes, technical lapses, inappropriate staffing in the recovery and ward areas, and greater reliance on residents. ${ }^{8,13,14,24}$

It is interesting to note that in our study, the highest crude in-hospital mortality was found in patients who underwent urgent or emergency surgery between 04:00 and 06:59, with a peak incidence between 04:00 and 04:59. This observation could relate to the fact that surgery commenced at 4.00 is driven by urgency and therefore risk; sicker patients are unable to wait. However, this could also be explained by unintentional overestimation of the risk of out of hours surgery by doctors, perhaps concealing an increase in risk-adjusted in-hospital mortality.

We did find that during the night, more patients who underwent emergency or urgent surgery were admitted to the ICU. In our opinion, this could be an indication that those patients are sicker and that the procedure they underwent could not be postponed until day-time. An alternative explanation for this finding is that there was no alternative to ICU admission because a suitable alternative care facility was unavailable. If so, it is not surprising that ICU admittance during the night was higher. Unfortunately, we do not have data available to study this alternative hypothesis. It could be that admission to an ICU during the night improved the outcome of those patients. If so, then perhaps the higher mortality due to nocturnal surgery is counterbalanced by an improved outcome for those admitted to the ICU during the night.

\section{Clinical implications}

Emergency surgery in sick patients need not wait until day-time, as mortality seems to be determined by factors related to the patient and surgical risk only, and not the time of day or seniority of the surgeon and anaesthesiologist performing the procedure. The impact of late night surgery falls on institutional resources. Staff who work at night need to rest the next day, possibly leading to cancelled cases or increased costs. This retrospective study is not designed to answer questions of this nature, but in future, studies with related outcomes are required. Our data indicate that the risk of being admitted to the ICU during the night after emergency and urgent noncardiac surgery is higher than during the day. This suggests that some organisation is required to have at least one bed available during the night.

\section{Limitations}

The present study has some obvious limitations. First, we retrospectively compared in-hospital mortality rates of patients undergoing emergency procedures during different time points of the day. Second, the parent study was not designed specifically to test this hypothesis. Without a biologically plausible and sound scientific rationale for the relationship between the variables (potential confounders) under study, the mortality and time of day, and 
having forced them into the model, some over fitting could have occurred. This could hide a true increased risk of mortality after procedures performed during the night. Third, we excluded all patients who underwent planned day-case surgery, cardiac surgery, neurosurgery, radiological or obstetric procedures. It could be that mortality is not evenly distributed across all patient groups and that by excluding certain patient groups, we also excluded evidence of a difference between times of surgery. Fourth, the assumption that the start time of the procedure is a proxy for team fatigue is only reasonable if the evening and night teams worked all day before their evening or night shifts. If those teams did not work in the day shift, then start time would be not the most valid measure. Our data did not include the daytime activities of the night shift, and therefore, we are unable to explore the exact role of fatigue in a potentially increased nocturnal mortality.

Fifth, the nature of the data collection could have introduced bias. Those patients undergoing high-risk surgery might have assumed a higher profile than those undergoing more mundane urgent and emergency procedures, and may have been overrepresented in data collection. Although we planned to enrol every eligible patient undergoing surgery during the study period, we cannot be sure of the exact proportion of eligible patients included. Sixth, there could be residual confounding, as 30-day mortality is not only influenced by the procedure or the time of the day on which the procedure took place but also by numerous known or unknown events or factors that took place between the procedure and the death of the patient. In our study, we do not have the details of what happened in this period, and are thus unable to study those factors. It may be that these aspects affected all three groups of patients in a similar fashion and did not interfere with our analysis. Finally, our study was underpowered to detect a marginal increase in risk-adjusted mortality. A $20 \%$ increase in mortality would be clinically very important, but would not have achieved statistical significance in the present analysis. A posthoc sample size calculation based on our results (daytime incidence $5.3 \%$, significance level 0.05 , power 0.80 ) shows that one would need about 17500 patients to reach statistical significance for the difference we have found. To overcome this limitation, a larger prospective study designed to address this specific question would be required. If this was a traditional cohort study, many centres and a considerable time would be needed to complete it. Our suggestion is to perform a casecontrol study in which patients who are operated on during the night (the cases) are compared with a sample of the patients operated on during the day (the controls). By using a case-control design, the number needed is reduced, and the study becomes more efficient.

\section{Conclusion}

We failed to find a relationship between in-hospital mortality and the time of day a procedure was performed in patients undergoing nonelective urgent or emergency surgery. This analysis would not have detected a small, in the region of $20 \%$, but nevertheless a clinically significant increase in mortality, and a larger prospective study is required.

\section{Acknowledgements relating to this article}

Assistance with the article: none.

Financial support: this work was supported by the European Society of Intensive Care Medicine, Brussels, Belgium, and the European Society of Anaesthesiology, Brussels, Belgium.

Conflict of interest: none.

Presentation: preliminary data for this study were presented as a poster presentation at the annual ESICM-meeting in Paris, September 2013.

\section{References}

1 Weiser TG, Regenbogen SE, Thompson KD, et al. An estimation of the global volume of surgery: a modelling strategy based on available data. Lancet 2008; 372:139-144.

2 Pearse RM, Moreno RP, Bauer P, et al. Mortality after surgery in Europe: a 7 day cohort study. Lancet 2012; 380:1059-1065.

3 Rhodes A, Moreno RP, Metnitz B, et al. Epidemiology and outcome following postsurgical admission to critical care. Intensive Care Med 2011; 37:1466-1472.

4 Pearse RM, Harrison DA, James $\mathrm{P}$, et al. Identification and characterisation of the high-risk surgical population in the United Kingdom. Crit Care 2006; 10:R81.

5 Jhanji S, Thomas B, Ely A, et al. Mortality and utilisation of critical care resources amongst high-risk surgical patients in a large NHS trust. Anaesthesia 2008; 63:695-700.

6 Glaser R, Naidu SS, Selzer F, et al. Factors associated with poorer prognosis for patients undergoing primary percutaneous coronary intervention during off-hours: biology or systems failure? JACC Cardiovasc Interv 2008; 1:681-688.

7 Ricci WM, Gallagher B, Brandt A, et al. Is after-hours orthopaedic surgery associated with adverse outcomes? A prospective comparative study. $J$ Bone Joint Surg Am 2009; 91:2067-2072.

8 Taffinder NJ, McManus IC, Gul Y, et al. Effect of sleep deprivation on surgeons' dexterity on laparoscopy simulator. Lancet 1998; 352:1191.

9 Tøttrup A, Erichsen R, Sværke C, et al. Thirty-day mortality after elective and emergency total colectomy in Danish patients with inflammatory bowel disease: a population-based nationwide cohort study. BMJ Open 2012; 2:e000823.

10 Guevara OA, Rubio-Romero JA, Ruiz-Parra AI. Unplanned reoperations: is emergency surgery a risk factor? A cohort study. J Surg Res 2013; 182:11-16.

11 Peets A, Ayas NT. Restricting resident work hours: the good, the bad, and the ugly. Crit Care Med 2012; 40:960-966.

12 Iglehart JK. Revisiting duty-hour limits - IOM recommendations for patient safety and resident education. N Engl J Med 2008; 359:2633-2635.

13 Komen N, Dijk J-W, Lalmahomed Z, et al. After-hours colorectal surgery: a risk factor for anastomotic leakage. Int J Colorectal Dis 2009; 24:789795.

14 Lonze BE, Parsikia A, Feyssa EL, et al. Operative start times and complications after liver transplantation. Am J Transplant 2010; 10:18421849.

15 Pearse RM, Rhodes A, Moreno R, et al. EuSOS: European surgical outcomes study. Eur J Anaesthesiol 2011; 28:454-456.

16 Anesthesiologists ASO. New classification of physical status. Anesthesiology 1963; 24:111.

17 Altman DG. Chapter 12: relation between several variables. Practical statistics for medical research. London: Chapmann \& Hall; 1990. pp. 325364.

18 George TJ, Arnaoutakis GJ, Merlo CA, et al. Association of operative time of day with outcomes after thoracic organ transplant. JAMA 2011; 305:2193-2199.

19 Katz JD. Issues of concern for the aging anesthesiologist. Anesth Analg 2001; 92:1487-1492.

20 Travis KW, Mihevc NT, Orkin FK, Zeitlin GL. Age and anesthetic practice: a regional perspective. J Clin Anesth 1999; 11:175-186. 
21 Vinden C, Nash DM, Rangrej J, et al. Complications of daytime elective laparoscopic cholecystectomies performed by surgeons who operated the night before. JAMA 2013; 310:1837-1841.

22 Fechner G, Pezold C, Hauser S, et al. Kidney's nightshift, kidney's nightmare? Comparison of daylight and nighttime kidney transplantation: impact on complications and graft survival. Transplant Proc 2008; $40: 1341-1344$.
23 Wright MC, Phillips-Bute B, Mark JB, et al. Time of day effects on the incidence of anesthetic adverse events. Qual Saf Health Care 2006; 15:258-263.

24 Grantcharov TP, Bardram L, Funch-Jensen P, Rosenberg J. Laparoscopic performance after one night on call in a surgical department: prospective study. BMJ 2001; 323:1222-1223. 